\title{
Review:
}

\section{Applications of advanced control methods in spacecrafts: progress, challenges, and future prospects*}

\author{
Yong-chun XIE ${ }^{\dagger 1,2}$, Huang HUANG ${ }^{\dagger 1,2}$, Yong HU ${ }^{1,2}$, Guo-qi ZHANG ${ }^{1,2}$ \\ $\left({ }^{1}\right.$ Science and Technology on Space Intelligent Control Laboratory, Beijing 100190, China) \\ ( ${ }^{2}$ Beijing Institute of Control Engineering, Beijing 100190, China) \\ †E-mail: xieyongchun@vip.sina.com; hhuang33@163.com \\ Received Mar. 7, 2016; Revision accepted June 24, 2016; Crosschecked Aug. 16, 2016
}

\begin{abstract}
We aim at examining the current status of advanced control methods in spacecrafts from an engineer's perspective. Instead of reviewing all the fancy theoretical results in advanced control for aerospace vehicles, the focus is on the advanced control methods that have been practically applied to spacecrafts during flight tests, or have been tested in real time on ground facilities and general testbeds/simulators built with actual flight data. The aim is to provide engineers with all the possible control laws that are readily available rather than those that are tested only in the laboratory at the moment. It turns out that despite the blooming developments of modern control theories, most of them have various limitations, which stop them from being practically applied to spacecrafts. There are a limited number of spacecrafts that are controlled by advanced control methods, among which $H_{2} / H_{\infty}$ robust control is the most popular method to deal with flexible structures, adaptive control is commonly used to deal with model/parameter uncertainty, and the linear quadratic regulator (LQR) is the most frequently used method in case of optimal control. It is hoped that this review paper will enlighten aerospace engineers who hold an open mind about advanced control methods, as well as scholars who are enthusiastic about engineering-oriented problems.
\end{abstract}

Key words: Spacecraft control, Robust control, Adaptive control, Optimal control http://dx.doi.org/10.1631/FITEE.1601063

CLC number: V448.22; TP273

\section{Introduction}

In aerospace engineering, reliability probably has the highest priority over many other criteria. System engineers usually are risk-adverse and adopt methods which have already been verified practically. Despite the blooming development of modern control theories, it is indisputable that the classical proportional-integral-derivative (PID) control still plays the dominant role in aerospace engineering. Since 1957, nearly 7800 spacecrafts have been launched, among which more than $99 \%$ used PID as

\footnotetext{
$\ddagger$ Corresponding author

* Project supported by the National Natural Science Foundation of China (Nos. 61203075, 61333008, and 61304027) and China Ministry of Science and Technology (No. 2013CB733100)

(B) ORCID: Yong-chun XIE, http://orcid.org/0000-0003-1412-0495 C)Zhejiang University and Springer-Verlag Berlin Heidelberg 2016
}

the baseline controller. Indeed, the already launched satellites or spacecrafts have testified that PID control can meet most of the fundamental mission requirements. Upon the baseline PID controller, various techniques, such as structure-bending filters and gain scheduling, have been designed to compensate for the lack of robustness and adaptability of the baseline controller. The control systems therefore become so complex that they lack flexibility and portability. On the other hand, with the increasing requirements of space exploration, the spacecrafts not only tend to exhibit different features, such as high flexibility, high-frequency oscillations, or unknown dynamics, but also face ultimate high-level performance requirements. This trend challenges the baseline PID controller and pushes engineers to advanced control methods. According to Hanson 
(2002), from 1991 to 2001, among all the launched vehicles by U.S., Europe, Japan, and Russia (that are involved with U.S. companies), $41 \%$ of the launch vehicle failures could be avoided by some advanced guidance and control where the control system would react quickly to failures and adjust its control parameters autonomously for different scenarios, instead of being tuned from the ground.

On the other hand, members of the control community are quite enthusiastic about developing novel and fancy control methods, most of which are motivated by the requirements on better robustness, more powerful adaptability, and higher-level system performance. There are millions of peer-reviewed reports and simulations demonstrating quite satisfactory outputs of those advanced control methods. In chemical engineering, apart from PID control, model predictive control (MPC) has become universal and has already been successfully applied to quite a few product lines. In aerospace engineering, there are robust control that originated in the 1970s and adaptive control that showed its potential as early as in the 1960s. However, in the 21st century, more than 99\% launched spacecrafts are still using PID. What is stopping us?

We believe that there are probably three reasons:

\section{Lack of a control-oriented model}

For spacecrafts, the Newton theory, the Euler theory, and the Kepler theory together ideally illustrate the kinetic and kinematic dynamics in a precise way. The corresponding mathematical models are characterized by multiple variables, high nonlinearities, and strong couplings. The unknown parameters determined by the mass, inertial, structure, and working status are difficult to measure because of the lack of high fidelity ground tests. Moreover, external disturbance and structure uncertainties are usually generated from experienced data, which cover only a limited number of working conditions. Therefore, controller design has to deal with unknown or changing parameters. Meanwhile, even when the dynamical model is precise and rigorous, it is usually highly nonlinear, highly ordered, and strongly coupled, and thus has to be simplified by techniques, such as linearization around trim conditions or model order reduction, so as to facilitate controller design.

2. Lack of perspective from engineers

Scholars are enthusiastic about getting motiva- tions from practical systems. However, when solving the mathematical problems generated from those motivations, various assumptions or constraints have to be made for a rigorous proof, e.g., the tuning of initial values in adaptive control and a known parameter bound in robust control. Although theoretically sound, those assumptions are usually impossible to meet in practice. In other words, theoretical results are usually more or less far from practice due to the various assumptions and constraints, and thus are difficult to accept and implement by engineers.

Moreover, taking adaptive control as an example, it can modify its parameters online so as to fit the changing environment and unmodeled dynamics. This nature makes adaptive control an ideal method in aerospace engineering where many uncertainties exist. However, adaptive control was never popular in the aerospace industry. The main reason is probably that system engineers without adaptive control background do not know where to start and how to start, while, on the other hand, an engineer can tune the parameters of a PID controller by following a list of specific guidelines without too much mathematical knowledge. Besides, engineers are used to judging a system according to analysis in the frequency domain. Once adaptive control is introduced, engineers will not be able to quantify the system performance in the frequency domain even if the design is carried out in this domain.

\section{Expense of computation}

One has to admit that PID is the simplest control algorithm requiring the least number of lines of codes and computation time. A complex algorithm not only increases the lines of codes but also brings huge troubles to system reliability. With the rapid development of hardware, the implementation of a complex algorithm may no longer be an issue, and hence the reliability.

To be more specific, in modern aerospace engineering, in both academia and industry, the main focuses in attitude control are high precision and fast maneuverability. With these increasing demands and the new generations of satellites, the main difficulties for spacecraft attitude control are as follows:

\section{Flexible structure}

The flexible mode of a spacecraft comes from its solar panels, antennas, flexible body, and sloshing effects in tanks during orbital maneuvers. It is difficult to build accurate dynamic models for these large 
flexible space structures on the ground due to the $1 \mathrm{~g}$ gravity and atmospheric effects, their low stiffness characteristics, etc., which lead to considerable modeling uncertainties established on the ground compared to the $0 \mathrm{~g}$ space environment. On-orbit system identification is a promising way that helps build an accurate model with identified parameters. This in turn makes the high-performance control design more feasible.

Even with an accurate model, controller design of the flexible structure is not easy. When the flexible structure is modeled as a distributed parameter system, currently the design of controller works only on simple sticks or boards and is not ready for engineers. By modeling the flexible structure as a centralized parameter model, various control methods including the classical one, the modern control theory, and the intelligent control theory have been studied by researchers.

\section{Unknown parameters}

The movement of spacecrafts in their orbit follows the basic physical rules, and most of them can be described by mathematical expressions. However, the space is full of mysteries, and it is almost impossible to anticipate all disturbance and uncertainties from ground experiments or simulations. Therefore, stability margin is one of the key indexes in PID controller design. When the true values are far from the ones used for simulation, the PID controller may fail to provide an adequate stability margin. As a controller with fixed parameters, robust control is the most commonly used method to deal with the unknown parameters. When the parameter uncertainties exceed the capability of robust control, adaptive control should be considered. The main issue is the implementation of those control methods in engineering practice. Engineers have to deal with the tuning in adaptive control and the calculation of parameter bounds through the entire flight regime.

\section{Changing parameters}

For the reentry of spacecrafts, the changes of atmosphere in terms of density, temperature, humidity, and ionosphere affect the internal and external model parameters. The changes of parameters usually extend to a level beyond the robustness of a PID controller. Robust control is ideal for such situations in which the parameters vary (fast or slow) within a limited domain. Adaptive control with online identification can deal with slowly time-varying parameters over a large range. When it comes to fast-changing parameters over large scales, currently there is no effective identification method. Multiple model adaptive control which switches between submodels was believed to be a promising way but was testified only through a limited number of numerical examples (Narendra and Han, 2011).

\section{High-level requirements}

High pointing accuracy, agile maneuverability, and minimum time/energy consumption are the high-level requirements in modern satellites. Theoretically, those requirements could be illustrated mathematically with mature solutions. However, for spacecrafts, various uncertainties and all kinds of constraints such as the allowed executing time, energy constraints, and actuator saturations would bring huge troubles during optimization. Meanwhile, computation complexity is another reason that stops most of the optimization methods from being applied in practice.

Despite all the obstacles that prevent advanced control methods from being applied in aerospace engineering, the increasing demands on modern spacecrafts have pushed engineers to embrace advanced control methods. NASA has long been interested in advanced control theories that can be applied in aerospace. Among those well-known modern control theories, adaptive control, neural network, and robust control are NASA's favorites; e.g., modelreference adaptive control has been proposed for highly accurate attitude control of satellites (Scarritt, 2008) and the $L_{1}$ adaptive feedback control has been presented for flexible wing (Cao and Hovakimyan, 2008; Kharisov et al., 2008).

In this review, we investigate a wide range of spacecrafts including satellites, the International Space Station (ISS), and reentry vehicles. The focus is on the advanced control laws that have been applied to spacecrafts with flight tests, or have been tested on ground facilities and general testbeds/simulators built with actual flight data. Meanwhile, to understand the effects of zero gravity, several middeck experiments have been conducted onboard the ISS to investigate the robustness and adaptability of different control methods, which are also included in this paper. The aim is to provide engineers with all the possible control algorithms that are readily available rather than those fancy control methods that are tested only within laboratory at 
the moment. We hope this material will enlighten aerospace engineers who hold an open mind about advanced control methods and scholars who are enthusiastic about engineering-oriented problems.

The rest of the paper is organized as follows. Section 2 summarizes the main advanced control laws that have been applied on-orbit or through ground tests with flight data. Those advanced control laws include optimal control, adaptive control, and robust control. In Section 3, four well-known satellites that use advanced control methods to suppress the flexible modes are introduced. Three of them are validated on-orbit and one of them with flight data. Section 4 focuses on the situation with unknown parameters, where identification and adaptive control algorithms are developed for two satellites and one onboard experiment. During the reentry of spacecrafts, the unknown and fast-changing environment poses a huge challenge to the control system. Much effort has been made to deal with the parameter changes, as introduced in Section 5 . Nowadays, high-performance requirements are required for spacecrafts, including ultra-high pointing accuracy, agile maneuver, and minimum reorientation time. Optimal control methods are introduced to meet those requirements, as shown in Section 6 . Finally, conclusions are drawn in Section 7, as well as future outlook.

\section{Advanced control methods}

\subsection{Linear quadratic regulator}

Linear quadratic regulator (LQR) is one of the optimal control methods that take the states of the dynamical system and control input into account (Antsaklis and Michel, 2007). By optimizing some criterion, closed-loop gains can be obtained. In general, the linear state-space equation can be obtained by linearizing the nonlinear system models around the equilibrium point:

$$
\dot{\boldsymbol{x}}=\boldsymbol{A} \boldsymbol{x}+\boldsymbol{B u},
$$

where $\boldsymbol{x} \in \mathbb{R}^{n}$ is the state vector, $\boldsymbol{u} \in \mathbb{R}^{m}$ is the input vector, and $\boldsymbol{A}$ and $\boldsymbol{B}$ are matrices with appropriate dimensions. The optimal state feedback control $\boldsymbol{u}=\boldsymbol{K} \boldsymbol{x}$ is derived by minimizing the following cost function:

$$
J=\int\left((\boldsymbol{x}(t))^{\mathrm{T}} \boldsymbol{Q} \boldsymbol{x}(t)+(\boldsymbol{u}(t))^{\mathrm{T}} \boldsymbol{R} \boldsymbol{u}(t)\right) \mathrm{d} t,
$$

where $\boldsymbol{Q}$ and $\boldsymbol{R}$ are positive definite matrices that give the compromise between the state transient energy and control input energy. The LQR gain matrix $\boldsymbol{K}$ is given by

$$
\boldsymbol{K}=\boldsymbol{R}^{-1} \boldsymbol{B}^{\mathrm{T}} \boldsymbol{P}
$$

where $\boldsymbol{P}$ is the unique positive definite solution to the following algebraic Riccati equation:

$$
\boldsymbol{A}^{\mathrm{T}} \boldsymbol{P}+\boldsymbol{P} \boldsymbol{A}-\boldsymbol{P} \boldsymbol{B} \boldsymbol{R}^{-1} \boldsymbol{B}^{\mathrm{T}} \boldsymbol{P}+\boldsymbol{Q}=\mathbf{0} .
$$

The typical use of the LQR problem is to determine the optimal control law $\boldsymbol{K}$ from a given set of weight matrices $\boldsymbol{Q}$ and $\boldsymbol{R}$.

\subsection{Inverse optimal control}

The direct method of designing a control law with good performance is to optimize an appropriate cost function. For most engineering applications, it is necessary to solve a Hamilton-Jacobi-Bellman (HJB) partial differential equation. The inverse optimal method offers a feasible approach to obtain an optimal feedback law (Freeman and Kokotovic, $1995 ; 1996)$. It is based on the fact that the solution to the appropriate HJB equation can be taken as a Lyapunov function that guarantees global stability.

The inverse optimal method avoids the monumental task of solving an HJB equation numerically. It consists of two basic stages. The first stage is to construct a stabilizing feedback controller called the benchmark controller. The controller is based on a control Lyapunov function. That is, there exists a proper and positive definite function $V$ for the following system:

$$
\dot{\boldsymbol{x}}=f(\boldsymbol{x})+g(\boldsymbol{x}) \boldsymbol{u}
$$

such that

$$
\inf _{\boldsymbol{u}}\left(L_{f}(V(\boldsymbol{x}))+L_{g}(V(\boldsymbol{x})) \boldsymbol{u}\right)<0,
$$

where $\boldsymbol{x} \in \mathbb{R}^{n}$ is the state vector, $\boldsymbol{u} \in \mathbb{R}^{m}$ is the control input, $f(\cdot), g(\cdot)$ are continuous functions, and $L_{f}(V(\boldsymbol{x})), L_{g}(V(\boldsymbol{x}))$ are the Lie derivatives of $V$ with respect to $f$ and $g$, respectively.

The second stage is to solve a nonlinear programming problem:

$$
\begin{cases}\min & J(\boldsymbol{u})=\boldsymbol{u}^{\mathrm{T}} \boldsymbol{u} \\ \text { s.t. } & L_{f}(V(\boldsymbol{x}))+L_{g}(V(\boldsymbol{x})) \boldsymbol{u} \leq-\sigma(\boldsymbol{x}) .\end{cases}
$$


The solution is

$$
\boldsymbol{u}^{*}=-\frac{\lambda}{2}\left[L_{g}(V(\boldsymbol{x}))\right]^{\mathrm{T}}
$$

where

$\lambda= \begin{cases}\frac{2\left(L_{f}(V(\boldsymbol{x}))+\sigma(\boldsymbol{x})\right)}{L_{g}(V(\boldsymbol{x}))\left[L_{g}(V(\boldsymbol{x}))\right]^{\mathrm{T}}}, & L_{f}(V(\boldsymbol{x}))>-\sigma(\boldsymbol{x}), \\ 0, & L_{f}(V(\boldsymbol{x})) \leq-\sigma(\boldsymbol{x}) .\end{cases}$

Then a minimum norm controller can be obtained (Bharadwaj et al., 1998).

\subsection{Pseudo spectral optimal control}

In recent years, pseudo spectral (PS) methods have been used to solve many nonlinear optimal control problems as introduced in Elnagar et al. (1995) and Ross and Fahroo (2004). The PS optimal control theory proposed by Ross and Karpenko (2012) is founded on the fact that any continuous function can be approximated to arbitrary precision by a polynomial, which is a direct consequence of the StoneWeierstrass approximation theorem (Rudin, 1975). A crucial question in implementation is how to select an appropriate polynomial basis and a computational grid. The most reliable computational grids used for spacecraft maneuvers are based on the Gauss-Lobatto (GL) points (Ross and Gong, 2010).

According to different polynomial bases and grids, various PS-based optimal control methods have been put forward to solve many practical problems in experimental demonstrations and flight operations (Ross and Karpenko, 2012). Usually, a practical optimal control problem requires full consideration of the nonlinearity and constraints, and can be defined as

$$
B:\left\{\begin{aligned}
\min \quad & J(\boldsymbol{x}, \boldsymbol{u})=E(\boldsymbol{x}(-1), \boldsymbol{x}(1)) \\
& +\int_{-1}^{1} F(\boldsymbol{x}(t), \boldsymbol{u}(t)) \mathrm{d} t \\
\text { s.t. } \quad & \dot{\boldsymbol{x}}(t)=f(\boldsymbol{x}(t), \boldsymbol{u}(t)), \\
& e(\boldsymbol{x}(-1), \boldsymbol{x}(1))=0, \\
& h(\boldsymbol{x}(t), \boldsymbol{u}(t)) \leq 0
\end{aligned}\right.
$$

where $F: \mathbb{R}^{N_{x}} \times \mathbb{R}^{N_{u}} \longrightarrow \mathbb{R}, E: \mathbb{R}^{N_{x}} \times \mathbb{R}^{N_{u}} \longrightarrow \mathbb{R}$, $f: \mathbb{R}^{N_{x}} \times \mathbb{R}^{N_{u}} \longrightarrow \mathbb{R}^{N_{x}}, e: \mathbb{R}^{N_{x}} \times \mathbb{R}^{N_{x}} \longrightarrow \mathbb{R}^{N_{e}}$, and $h: \mathbb{R}^{N_{x}} \times \mathbb{R}^{N_{x}} \longrightarrow \mathbb{R}^{N_{h}}$, with $N_{x}, N_{u}, N_{e}, N_{h}$ the corresponding dimensions.

Choose an arbitrary grid between the boundary points $t_{0}=-1$ and $t_{N}=1$, where $-1<t_{1}<t_{2}<$ $\ldots<t_{N-1}<1$. Using the GL points, the state function $\boldsymbol{x}(t)$ and control function $\boldsymbol{u}(t)$ can be approximated. By the differentiation and integration of the state and control functions, the path constraints are enforced only at the GL points, and the problem is then transformed to guarantee the values of the state and control at the GL points. Therefore, the problem $B$ can be transformed into a relative finite-dimensional problem $B^{\mathrm{N}}$ by PS discretization:

$$
B^{\mathrm{N}}:\left\{\begin{aligned}
\min \quad & J(\boldsymbol{x}, \boldsymbol{u})=E(\boldsymbol{x}(0), \boldsymbol{x}(N)) \\
& +\sum_{i=0}^{N} F(\boldsymbol{x}(i), \boldsymbol{u}(i)) \omega_{i} \\
\text { s.t. } \quad & \sum_{j=0}^{N} D_{i j} \boldsymbol{x}(j)=f(\boldsymbol{x}(i), \boldsymbol{u}(i)), \\
& e(\boldsymbol{x}(0), \boldsymbol{x}(N))=0 \\
& h(\boldsymbol{x}(i), \boldsymbol{u}(i)) \leq 0 \\
& i=0,1, \ldots, N,
\end{aligned}\right.
$$

where $\boldsymbol{D}$ is a square differentiation matrix and $\omega_{i}$ is the weight satisfying

$$
\begin{gathered}
D_{i j}=\dot{\phi}_{j}\left(t_{i}\right), \quad \omega_{i}=\int_{-1}^{1} \phi_{i}(t) \mathrm{d} t, \\
\phi_{i}(t)=\frac{g_{N}(t)}{g_{N}^{\prime}\left(t_{i}\right)\left(t-t_{i}\right)}, \quad g_{N}(t)=\prod_{i=0}^{N}\left(t-t_{i}\right) .
\end{gathered}
$$

The optimal control problem $B^{\mathrm{N}}$ is a nonlinear programming (NLP) problem. For smooth situations, as the number of GL points increases, the interpolation error decreases faster than the polynomial rates. PS methods are useful in practice because the optimality verification and validation for the solution can be quite readily done by the covector mapping theorem (Ross, 2005a; 2005b), and the feasibility and convergence can also be guaranteed (Gong et al., 2008). A MATLAB implementation of the PS method is DIDO, a PS-based optimal control solver package (Ross, 2007). The software acts much like a 'black-box', which allows a user to formulate an optimal control problem in m-code format. A Fortran implementation is available under OTIS by NASA (Paris et al., 2006).

\subsection{Model reference adaptive control}

Model reference adaptive control (MRAC), developed in the 1950s (Åström and Wittenmark, 2008), is a rigorous and systematic method in adaptive control. The basic principle is illustrated in Fig. 1. The main idea of MRAC is to make the 
output $\boldsymbol{y}$ of the plant track the output $\boldsymbol{y}_{\mathrm{r}}$ of a reference model defined beforehand by adjusting the controller parameters $\hat{\boldsymbol{\theta}}$. The reference model specifies the system performance and tells how to respond to the command signal $\boldsymbol{r}$. The adaptive law in the parameter adjustment block is used to update the parameters of the controller. The schematic diagram of the MRAC system basically consists of two loops: the first loop is normal feedback control, and the second loop is parameter adjustment. The main approaches to the analysis and design of the MRAC include the gradient approach, Lyapunov functions, and passivity theory.



Fig. 1 Block diagram of a model reference adaptive control system

Consider the following nonlinear plant:

$$
\dot{\boldsymbol{x}}(t)=\boldsymbol{A} \boldsymbol{x}(t)+\boldsymbol{B}(\boldsymbol{u}(t)+f(\boldsymbol{x}(t))),
$$

where $\boldsymbol{x}(t)$ is a state vector, $\boldsymbol{u}(t)$ is a control vector, $\boldsymbol{A}, \boldsymbol{B}$ are known and controllable, $f(\boldsymbol{x}(t))=$ $\boldsymbol{\theta}^{\mathrm{T}} \Phi(\boldsymbol{x}(t))+\varepsilon(\boldsymbol{x}(t))$ is an uncertain term which can be linearly approximated by a set of continuous, differentiable, and bounded basis functions $\Phi(\boldsymbol{x}(t))$, and $\varepsilon(\boldsymbol{x}(t))$ is an approximation error. The reference model can be written as

$$
\dot{\boldsymbol{x}}_{m}(t)=\boldsymbol{A}_{m} \boldsymbol{x}_{m}(t)+\boldsymbol{B}_{m} \boldsymbol{r}(t),
$$

where $\boldsymbol{x}_{m}(t)$ is a reference state vector, $\boldsymbol{r}(t)$ is a bounded piecewise continuous command vector, and $\boldsymbol{A}_{m}$ (Hurwitz) and $\boldsymbol{B}_{m}$ are matrices with appropriate dimensions. The aim is to design an adaptive controller to ensure $\boldsymbol{x}(t)$ to track $\boldsymbol{x}_{m}(t)$ :

$$
\boldsymbol{u}(t)=\boldsymbol{K}_{1} \boldsymbol{x}(t)+\boldsymbol{K}_{2} \boldsymbol{r}(t)-(\hat{\boldsymbol{\theta}}(t))^{\mathrm{T}} \Phi(\boldsymbol{x}(t)),
$$

where $\boldsymbol{K}_{1}$ and $\boldsymbol{K}_{2}$ are constant matrices and the last term is a direct adaptive signal. Note that $\hat{\boldsymbol{\theta}}$ is the estimated value of $\boldsymbol{\theta}$. Assume that there exist $\boldsymbol{K}_{1}$ and $\boldsymbol{K}_{2}$ such that $\boldsymbol{A}_{m}=\boldsymbol{A}+\boldsymbol{B} \boldsymbol{K}_{1}$ and $\boldsymbol{B}_{m}=\boldsymbol{B} \boldsymbol{K}_{2}$. Then the tracking error equation is as follows:

$$
\dot{\boldsymbol{e}}(t)=\boldsymbol{A}_{m} \boldsymbol{e}(t)+\boldsymbol{B}((\hat{\boldsymbol{\theta}}-\boldsymbol{\theta}) \Phi(\boldsymbol{x}(t))-\varepsilon(\boldsymbol{x}(t))) .
$$

The parameter update law which minimizes $\|\boldsymbol{e}(t)\|$ can be designed as

$\dot{\hat{\boldsymbol{\theta}}}=-\boldsymbol{\Gamma} \Phi(\boldsymbol{x}(t))\left((\boldsymbol{e}(t))^{\mathrm{T}} \boldsymbol{P}-v(\Phi(\boldsymbol{x}(t)))^{\mathrm{T}} \hat{\boldsymbol{\theta}} \boldsymbol{B}^{\mathrm{T}} \boldsymbol{P} \boldsymbol{A}_{m}^{-1}\right) \boldsymbol{B}$,

where $\boldsymbol{\Gamma}$ is a positive definite matrix, $v$ is a weighting constant, and $\boldsymbol{P}$ is obtained by solving the following Riccati equation:

$$
\boldsymbol{P} \boldsymbol{A}_{m}+\boldsymbol{A}_{m}^{\mathrm{T}} \boldsymbol{P}=-\boldsymbol{Q},
$$

where $\boldsymbol{Q}$ is a positive definite matrix. The uniformly ultimately bounded tracking error can be obtained by choosing a Lyapunov candidate function

$$
V(t)=(\boldsymbol{e}(t))^{\mathrm{T}} \boldsymbol{P} \boldsymbol{e}(t)+\operatorname{tr}\left((\hat{\boldsymbol{\theta}}-\boldsymbol{\theta})^{\mathrm{T}} \boldsymbol{\Gamma}(\hat{\boldsymbol{\theta}}-\boldsymbol{\theta})\right),
$$

where 'tr' denotes the trace operation. The detailed proof can be found in Burken et al. (2010).

\subsection{Characteristic model-based golden- section adaptive control}

Self-tuning is a classical adaptive control method that helps deal with time delay and disturbance. A self-tuning controller identifies the parameters according to the disturbed input and output, and generates control signals online.

As a special kind of self-tuning adaptive control, the characteristic model-based adaptive control method was first proposed by Wu (1990). It has received great development in theory and engineering applications over the last decade (Wu et al., 2009). The characteristic model was developed to use a loworder discrete time-varying system to deal with a high-order linear or nonlinear system, based on the dynamic characteristics of the plant and the required control performance. Rather than dropping information as in the reduced-order modeling, it compresses all the information of the high-order model into several characteristic parameters. The characteristic model is an online adaptive one so as to fit into the changing environment. Consider the general transfer function for a single-input single-output (SISO) linear time-invariant (LTI) system:

$$
G(s)=\frac{b_{m} s^{m}+b_{m-1} s^{m-1}+\ldots+b_{1} s+b_{0}}{s^{n}+a_{n-1} s^{n-1}+\ldots+a_{1} s+a_{0}},
$$

where $a_{i}(i=0,1, \ldots, n-1)$ and $b_{i}(i=$ $0,1, \ldots, m)$ are constant parameters.

When the control requirement is keeping or tracking a position, its characteristic model can be 
described by

$$
\begin{aligned}
\boldsymbol{y}(k+1)= & f_{1}(k) \boldsymbol{y}(k)+f_{2}(k) \boldsymbol{y}(k-1)+g_{0}(k) \boldsymbol{u}(k) \\
& +g_{1}(k) \boldsymbol{u}(k-1) .
\end{aligned}
$$

When the LTI system (1) is stable or contains integral components, we can see that (Wu et al., 2001): (1) the coefficients are slowly time-varying; (2) the range of the coefficients can be determined beforehand (Wu et al., 2009); (3) the output of the characteristic model becomes arbitrarily closer to that of the plant as the sampling period decreases; and (4) the sum of the coefficients at the steady state is equal to 1 if the static gain is 1 , i.e.,

$$
f_{1}(\infty)+f_{2}(\infty)+g_{0}(\infty)+g_{1}(\infty)=1 .
$$

In practice, we have $g_{0} \in[0.003,0.3]$ and $\left|g_{1}(k)\right| \leq$ $g_{0}(k)$. For a stable plant, if $T / T_{\min } \in[1 / 10,1 / 3]$, where $T_{\min }$ is the minimum equivalent time constant of the plant, the values of the characteristic parameters $f_{1}(k)$ and $f_{2}(k)$ belong to the following set:

$$
D_{\mathrm{S}}:\left\{\begin{array}{l}
f_{1} \in[1.4331,1.9974] \\
f_{2} \in[-0.9999,-0.5134] \\
f_{1}+f_{2} \in[0.9196,0.9999]
\end{array}\right.
$$

On the other hand, for an unstable plant, if $T / T_{\min } \in$ $[1 / 10,1 / 4]$, the values of the characteristic parameters $f_{1}(k)$ and $f_{2}(k)$ belong to the following set:

$$
D_{\mathrm{N}}:\left\{\begin{array}{l}
f_{1} \in[1.9844,2.2663], \\
f_{2} \in[-1.2840,-1], \\
f_{1}+f_{2} \in[0.9646,1] .
\end{array}\right.
$$

The characteristic parameters can be updated by the projected gradient algorithm as follows:

$$
\left\{\begin{aligned}
& \hat{\boldsymbol{\theta}}_{\boldsymbol{u}}(k)=\hat{\boldsymbol{\theta}}(k-1) \\
&+\frac{\gamma \boldsymbol{\phi}(k-1)\left(\boldsymbol{y}(k)-(\boldsymbol{\phi}(k-1))^{\mathrm{T}} \hat{\boldsymbol{\theta}}(k-1)\right)}{\delta+(\boldsymbol{\phi}(k-1))^{\mathrm{T}} \boldsymbol{\phi}(k-1)}, \\
& \hat{\boldsymbol{\theta}}(k)=\pi\left[\hat{\boldsymbol{\theta}}_{\boldsymbol{u}}(k)\right],
\end{aligned}\right.
$$

where

$$
\left\{\begin{array}{l}
\boldsymbol{\phi}(k)=[\boldsymbol{y}(k), \boldsymbol{y}(k-1), \boldsymbol{u}(k), \boldsymbol{u}(k-1)]^{\mathrm{T}}, \\
\boldsymbol{\theta}(k)=\left[f_{1}(k), f_{2}(k), g_{0}(k), g_{1}(k)\right]^{\mathrm{T}}, \\
\hat{\boldsymbol{\theta}}(k)=\left[\hat{f}_{1}(k), \hat{f}_{2}(k), \hat{g}_{0}(k), \hat{g}_{1}(k)\right]^{\mathrm{T}},
\end{array}\right.
$$

$\delta>0,0<\gamma<2$ are constants, and $\pi[\cdot]$ is the orthogonal projector.
The estimated parameters are constrained within the convex domain $D_{\mathrm{S}}$ or $D_{\mathrm{N}}$.

The characteristic model-based golden-section adaptive control $\boldsymbol{u}(k)$ is formulated as (Wu et al., 2009)

$$
\begin{aligned}
\boldsymbol{u}(k)= & \frac{1}{\hat{g}_{0}(k)+\lambda}\left(l_{1} \hat{f}_{1}(k) \boldsymbol{e}(k)+l_{2} \hat{f}_{2}(k) \boldsymbol{e}(k-1)\right. \\
& \left.-\hat{g}_{1}(k) \boldsymbol{u}(k-1)\right),
\end{aligned}
$$

where $\boldsymbol{e}(k)=\boldsymbol{y}_{\mathrm{r}}(k)-\boldsymbol{y}(k), l_{1}=0.382$, and $l_{2}=$ 0.618. The robust stability of the golden-section adaptive control law was proved in Xie and $\mathrm{Wu}$ (1992) and Huang (2015).

The golden-section controller is simple and easy to apply in practice. Over the past 20 years, this control scheme has been applied to more than 400 systems belonging to 10 kinds of engineering plants in the fields of astronautics (such as in the successful rendezvous and docking of Shenzhou-8 spacecraft (Hu et al., 2011) and reentry adaptive control of Shenzhou spacecraft (Hu, 1998)) and industry in China (Wu et al., 2007).

\section{$2.6 H_{\infty}$ and $H_{2}$ control}

Consider a generalized system (Fig. 2), where $\boldsymbol{G}$ and $\boldsymbol{K}$ are real, rational, and proper. Assume that the state-space representations of $\boldsymbol{G}$ and $\boldsymbol{K}$ are controllable and observable. The exogenous input $\boldsymbol{w}$ could be disturbance, sensor noise, or commands, $\boldsymbol{z}$ is the controlled output, and $\boldsymbol{v}$ is the measured output. The closed-loop transfer function from $\boldsymbol{w}$ to $\boldsymbol{z}$ is denoted by $\boldsymbol{T}_{\boldsymbol{w} \boldsymbol{z}}$ whose $H_{\infty}$ norm is

$$
\left\|\boldsymbol{T}_{\boldsymbol{w} \boldsymbol{z}}\right\|_{\infty}=\sup _{\boldsymbol{w}} \bar{\sigma}\left(\boldsymbol{T}_{\boldsymbol{z w}}(\mathrm{j} \boldsymbol{w})\right)
$$

The $H_{\infty}$ optimal control is to find a controller $\boldsymbol{K}$ such that the $H_{\infty}$ norm of $\boldsymbol{T}_{\boldsymbol{w} \boldsymbol{z}}$ is minimized (Zhou et al., 1996). A smaller $H_{\infty}$ norm indicates that the exogenous input $\boldsymbol{w}$ has less disturbance on the output $\boldsymbol{z}$, meaning the closed-loop system has stronger robustness to disturbance.

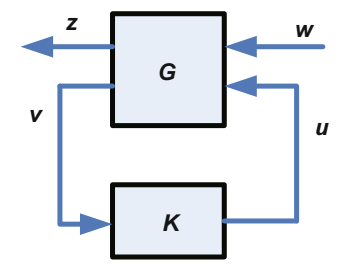

Fig. 2 Generalized plant and controller configuration 
In the more generalized case, we are interested in the suboptimal problem by finding $\boldsymbol{K}$ such that $\left\|\boldsymbol{T}_{\boldsymbol{w} \boldsymbol{z}}\right\|_{\infty}<\gamma$.

Once the suboptimal $H_{\infty}$ controller $\boldsymbol{K}$ is found, the system in Fig. 3 is stable for all admissible uncertainty $\|\boldsymbol{\Delta}\|_{\infty}<1 / \gamma$.

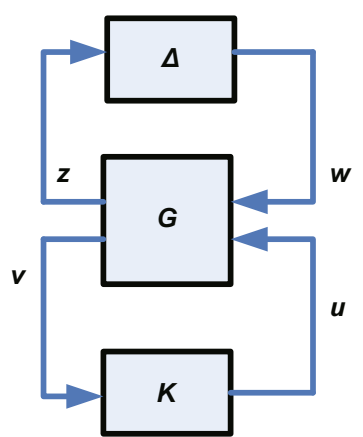

Fig. 3 Generalized plant and controller configuration with disturbance

The $H_{2}$ optimal and suboptimal problems are similar to the $H_{\infty}$ problem, with the only difference that the norm of the transfer function is calculated according to

$$
\left\|\boldsymbol{T}_{\boldsymbol{w} \boldsymbol{z}}\right\|_{2}=\operatorname{tr}\left(\frac{1}{2 \pi} \int_{-\infty}^{\infty} \boldsymbol{T}(\mathrm{j} \boldsymbol{w}) \boldsymbol{T}^{*}(\mathrm{j} \boldsymbol{w}) \mathrm{d} \boldsymbol{w}\right)^{1 / 2}
$$

where ${ }^{* *}$ represents the conjugate transpose.

The synthesis of the $H_{\infty}$ controller $\boldsymbol{K}$ is wellposed. The original way is to use the NevanlinnaPick interpolating method or the operator method, which met with many problems when dealing with the multi-input multi-output (MIMO) system. Therefore, modern $H_{\infty}$ control theory was built upon the state-space method (Doyle, 1984; Glover, 1984; Francis, 1987). In the $1980 \mathrm{~s}$ and $1990 \mathrm{~s}, H_{\infty}$ control theory experienced blooming development theoretically and practically. Readers are referred to $\mathrm{Yu}$ (2002) for the linear matrix inequality (LMI) based synthesis of those controllers. The LMI toolbox released by MATLAB in 1995 further provided a handy way to synthesize $H_{\infty} / H_{2}$ controllers.

\section{$2.7 \mu$ synthesis}

The definition of $\mu$ is motivated by finding the smallest destabilizing matrix $\boldsymbol{\Delta}$ to a given matrix $\boldsymbol{M}$. The solution is

$$
\boldsymbol{\Delta}_{d}=\frac{1}{\bar{\sigma}(\boldsymbol{M})} \boldsymbol{v}_{1} \boldsymbol{u}_{1}^{*}
$$

where $\boldsymbol{M}=\bar{\sigma}(\boldsymbol{M}) \boldsymbol{u}_{1} \boldsymbol{v}_{1}^{*}+\sigma_{2} \boldsymbol{u}_{2} \boldsymbol{v}_{2}^{*}+\ldots$ is a singular value decomposition.

Suppose $\Delta$ is structured by

$$
\begin{aligned}
\overline{\boldsymbol{\Delta}}= & \left\{\operatorname { d i a g } \left(\delta_{1} \boldsymbol{I}_{r_{1}}, \delta_{2} \boldsymbol{I}_{r_{2}}, \ldots, \delta_{S} \boldsymbol{I}_{r_{S}}, \overline{\boldsymbol{\Delta}}_{1}, \overline{\boldsymbol{\Delta}}_{2}, \ldots,\right.\right. \\
& \left.\left.\overline{\boldsymbol{\Delta}}_{F}\right): \delta_{i} \in \mathbb{C}, \overline{\boldsymbol{\Delta}}_{j} \in \mathbb{C}^{m_{j} \times m_{j}}\right\},
\end{aligned}
$$

where $S$ is the number of repeated scalar blocks and $F$ is the number of full blocks of the block diagonal matrix $\boldsymbol{\Delta}$. When $\boldsymbol{\Delta}$ is block diagonalized by $\bar{\Delta}$, the smallest perturbation matrix that destabilizes $\boldsymbol{M}$ is Eq. (3) with

$\mu_{\overline{\boldsymbol{\Delta}}}(\boldsymbol{M}):=\frac{1}{\min \{\bar{\sigma}(\boldsymbol{\Delta}): \boldsymbol{\Delta} \in \overline{\boldsymbol{\Delta}}, \operatorname{det}(\boldsymbol{I}-\boldsymbol{M} \boldsymbol{\Delta})=0\}}$.

If no $\boldsymbol{\Delta} \in \overline{\boldsymbol{\Delta}}$ makes $\boldsymbol{I}-\boldsymbol{M} \boldsymbol{\Delta}$ singular, then $\mu_{\bar{\Delta}}(\boldsymbol{M}):=0$.

The $\mu$ stability of the system in Fig. 4 is given by the following lemma (Zhou and Doyle, 1999):

Lemma 1 The system consisting of $\boldsymbol{M}$ and $\boldsymbol{\Delta}$ is well-posed, internally stable, and $\left\|\boldsymbol{T}_{\boldsymbol{w} \boldsymbol{z}}\right\|_{\infty} \leq \beta$ for all $\boldsymbol{\Delta}(s) \in \mathcal{M}(\overline{\boldsymbol{\Delta}})$ with $\|\overline{\boldsymbol{\Delta}}\|_{\infty}<1 / \beta$ if and only if

$$
\sup _{w \in \mathbb{R}} \mu_{\overline{\boldsymbol{\Delta}}_{P}}(\boldsymbol{G}(\mathrm{j} w)) \leq \beta,
$$

where $\mathcal{M}(\overline{\boldsymbol{\Delta}}):=\left\{\boldsymbol{\Delta}(\cdot) \in \mathcal{R H}_{\infty}: \boldsymbol{\Delta}(s) \in \overline{\boldsymbol{\Delta}}, \forall s \in\right.$ $\left.\overline{\mathbb{C}}_{+}\right\}$.

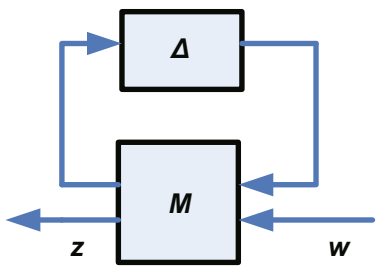

Fig. 4 System framework

\section{Orbital spacecraft with flexible structure}

Flexibility is probably the biggest challenge in spacecraft control. Flexibility comes from large solar panels, antennas, and the sloshing effects in tanks. A flexible structure may produce large structure vibrations, which are modeled by a high-order equation with an infinite number of modes acting over a wide frequency range. Because of the lack of experimental data at zero gravity, a flexible structure also brings parameter uncertainties. Meanwhile, the solar panels are usually light-weight with small damping ratios and can be excited easily. 
The modeling of a spacecraft with a flexible structure aims to build the coupling equation concerning the movements of the spacecraft and structure vibrations. In most cases, the flexible structures are modeled by the finite element analysis method, and then mixed together into the overall model based on the Lagrange equations. The order of the model has to be reduced so as to facilitate controller design.

In many applications, the traditional PID control has been proved to be short-handed. In this section, we find as many as three kinds of spacecraft that used advanced control methods to deal with the flexibility, all of which were tested on-orbit. The wellknown Hubble Space Telescope is also introduced in the end. Its advanced control experiment was carried out on the ground but with actual flight data.

\subsection{Spacebus 4000 telecommunication satel- lite}

Thales Alenia Space-France (TAS-F) has developed a geosynchronous telecommunication platform named Spacebus 4000, which has been applied to 15 telecommunication satellites with satisfactory performance. Telecommunication satellites are characterized by distinctive flexibilities due to their moving appendages including solar arrays, antennas, and sloshing effects in tanks. According to reports, those flexible modes are badly damped at 0.001 with uncertain frequencies at $\pm 30 \%$. To deal with the flexibility, a conventional way is to design some filters with carefully tuned parameters, together with the baseline PID controller, so as to attenuate its resonance. For example, after the deployment of the solar array on the ISS, unexpected flexibility problems were observed, and two filters were added so as to accomplish the reorientation maneuver on-orbit.

TAS-F sponsored research on $H_{\infty}$ controller synthesis to deal with the flexibility. The control system structure on Spacebus 4000 was presented by Charbonnel (2010).

The $H_{\infty}$ synthesis is to find a controller $\boldsymbol{K}(s)$ to the following optimization problem:

$$
\min \left\|G\left(\left[\begin{array}{l}
r \\
d
\end{array}\right] \rightarrow\left[\begin{array}{l}
e_{1} \\
e_{2}
\end{array}\right]\right)\right\|_{\infty},
$$

where $G(x \rightarrow y)$ is the transfer function from signal $x$ to signal $y, r$ is the reference, $d$ is the disturbance, and $e_{i}(i=1,2)$ is the output. A low-pass filter and a high-pass filter are introduced to the outputs, and a disturbance rejection filter is designed for $d$. The stability is guaranteed by the $H_{\infty}$ controller, and thus the tuning of the above filters is much relaxed. One of the key technologies is to solve the optimization problem. Because of numerical issues and conservative solutions, the Riccati equation based Glover-Doyle algorithm is used instead of the LMI method. Meanwhile, the design of the controller is a worst case model with flexible mode frequencies, being close to the control bandwidth so as to improve the robustness. The delays are treated as first-order approximations during the controller design.

The $H_{\infty}$ controller was compared with the classical PID controller on the TAS-F high-fidelity simulator AOCS (attitude and orbit control system). The $H_{\infty}$ controller showed improved stability margins, stronger robustness, better dynamic performance, and less fuel consumption than the PID controller during orbit correction maneuvers.

The $H_{\infty}$ approach is the core control algorithm of the Spacebus 4000 platform, which is now the TAS-F industrial baseline. Since 2003, it has been serving 15 telecommunication satellites with excellent pointing accuracy and stability. It is worth mentioning that the entire design process is impressively engineer-friendly. Half a day's training session would allow any engineer to design the controller.

\subsection{Engineering Test Satellite-VI/VIII}

The Engineering Test Satellite-VI (ETS-VI) was initiated by the National Space Development Agency of Japan (NASDA). It is a three-axis stabilized geosynchronous spacecraft with a pair of large lightweight solar panels. The mission of this satellite is to execute advanced communication experiments. It is a challenge to control such a large flexible spacecraft with high accuracy due to its structure vibration and control-structure interactions.

ETS-VI was launched in Aug. 1994. After the launch, NASDA carried out on-orbit system identification experiments from Dec. 1994 to Mar. 1995 to check the validity of the model for ETS-VI obtained from ground experiments. In the system identification experiments, the attitude angle and rate of the satellite's main body, the thruster drive signals, and the precise accelerometer (PACC) signals were used as the measurements for two system identification methods, which are the traditional methods based on the polynomial black-box models and a 
subspace-based method. Two forms of excitations were used as the inputs to the spacecraft: impulse excitations and random excitations. The results showed that both methods constructed an accurate mathematical model of the satellite (Adachi et al., 1999).

During controller design, the full-order model was reduced to three low-order ones according to different vibration modes. Two types of continuous time linear controllers, which were discretized at the sampling rate of $4 \mathrm{~Hz}$ during implementation, were developed to achieve robust stability against residual modes and modal parameter errors. The first type is a frequency-dependent LQR with a state estimator. This type of controller has robustness against the residual modes. The second type is an $H_{\infty}$ controller. The original $H_{\infty}$ control approach could be ill-conditioned when dealing with the lightly damped vibration modes and the undamped rigid modes simultaneously. Therefore, two kinds of methods, namely the robust stability degree assignment method and the direct velocity feedback method, were employed during the $H_{\infty}$ design so as to deal with the zero poles in the reduced model. To evaluate the identified model and the controllers, on-orbit experiments were carried out after the launch in 1995 . The designed controllers held the panel rotation at the angle of $270^{\circ}$ or $180^{\circ}$ according to the experimental data. Step response and impulse response of the attitude control to the disturbance torque were tested to evaluate the controller performance. The results confirmed the validity of the LQR and the $H_{\infty}$ design methods based on the reduced-order model, and the ability of these control methods with highfrequency residual modes and parameter uncertainties. Besides, online identification was carried out by vibrating the spacecraft with the pseudo-random and the impulse signals (Kida et al., 1997).

The ETS-VIII, launched in Dec. 2006, is the largest satellite developed by Japan to date, with a size of $40 \mathrm{~m} \times 37 \mathrm{~m}$ and a mass of $3000 \mathrm{~kg}$. The spacecraft has two large deployable flexible reflectors and two flexible solar panels that rotate around the pitch axis, which makes dynamic coupling between the three axes, and the system parameters change by $25 \%$ at the maximum according to the paddle rotation angle. To guarantee robust stability of the attitude control system for the MIMO linear-parametervarying (LPV) system against the higher vibration modes and model parameter uncertainties, four new control methods were designed and examined onorbit, namely the $\mu$-synthesis linear time-invariant controller (Ohtani et al., 2009), the interpolationbased gain scheduling controller (Hamada et al., 2011), the 2-DOF (degree-of-freedom) static output feedback controller, and the 2-DOF dynamic output feedback controller (Nagashio et al., 2014). A baseline PD controller for the flight test was used to be compared with these control methods. From June 2009 to Mar. 2010, Japan carried out several orbital experiments to testify the dynamic performance with step response, impulse disturbance, square disturbance, and random disturbance. Compared to the traditional PD controller, the advanced controllers can achieve superior performance.

\subsection{Hubble Space Telescope}

In Apr. 1990, the United States launched the Hubble Space Telescope (HST) to a 611-km circular orbit. Two solar arrays and two high-gain antennas were deployed on the telescope. When analyzing the flight data, the engineers noticed that, due to daynight changes, the thermally induced deformations of the solar arrays caused unexpectedly large perturbations in the pointing control system. The baseline PID controller of the pointing control system was unable to deal with such perturbations. Much effort was made from both modeling and control perspectives. First, several parameters were collected from two on-orbit identification experiments to identify the disturbance frequencies and the three-axis system transfer functions. Frequency identification techniques were used in the two experiments including the fast Fourier transforms (FFTs) and spectral analysis of the measurements on the reaction wheel assemblies (RWAs) and the rate gyro assembly (RGA). Through the identifications, more accurate transfer functions were obtained (Anthony and Andersen, 1995). Second, the engineers designed two sixth-order filters to reshape the disturbance rejection transfer function such that the disturbance was attenuated when it entered the dominant solar array frequencies. Although the modified pointing control system provided superb pointing accuracy, the tuning of the filters was quite tricky, and depended heavily on the flight data, which was an afterward remedy and could not be replicated (Nurre et al., 1995). 
To avoid similar problems in the later missions, several advanced modern control methodologies were developed and tested using actual flight data collected from the HST. The research was sponsored by the Marshall Space Flight Center. Five modern control strategies were conducted by five different research groups. The University of Colorado in Boulder studied the problem based on the disturbanceaccommodating control (DAC) with a reduced-order model (ROM) controller, a disturbance estimator, and a residual mode filter. The Harris Corporation team solved the problem with the linear quadratic Gaussian (LQG) approach. The Ohio University design team proposed an $H_{\infty}$ design method. The Purdue University team designed two variations of covariance controllers. The University of Alabama in Huntsville (UAH) research group designed total isolation (TI) and array damping (AD) strategies to deal with solar array disturbance (Bukley, 1995). These five research groups testified their control strategies in different ways. The ROM-based DAC controller showed promising results in the linear MIMO model with quite satisfactory pointing errors. The tracking performance of the LQG method outperformed that of the PID controller being deployed onboard. An 82nd-order $H_{\infty}$ controller design was obtained using the MIMO modal plant model at $90^{\circ}$. However, this controller worked only for this specific model. Therefore, an improved $H_{\infty}$ design method was further developed that resulted in improved performance in peak and attitude errors. The covariance controller was evaluated on the 83rdorder actual model. One improvement of this control law was the control energy, which was significantly less than that of the original controller onboard. Finally, UAH developed a planar simulation that included all the main body and solar array interface dynamics of the HST, and testified its TI and AD controllers in this simulation. HST with the TI controller can maintain the pointing stability despite the solar array vibrations. However, the stability margin and robustness to parameter uncertainty of the AD controller were unsatisfactory.

\section{Spacecraft with unknown parameters}

When parameter variation exceeds the robustness of the PID controller, advanced control methods have to be considered. In this section, the Shenzhou reentry spacecraft from China and the Data Relay Test Satellite (DRTS) from Japan are introduced. The United States has also conducted a middeck experiment onboard the Space Shuttle, which is presented in the end.

\subsection{Shenzhou spacecraft}

The research on rendezvous and docking (RVD) technology in China began in the late part of the last century. Automatic control and manual control were developed at the same time. China's first RVD operation was achieved in Nov. 2011 between spacecrafts Shenzhou-8 and Tiangong-1 (Hu et al., 2011). In the next year, the Chinese first manual RVD task was also successfully completed by Shenzhou-9 and Tiangong-1 in June 2012 (Xie et al., 2013).

Shenzhou spacecraft is composed of an orbital module, a reentry module, and a propulsion module. There are two large solar panels on the propulsion module. During RVD, attitude maneuvers and orbit control are frequently performed by firing the appropriate pairs of thrusters. This excites the flexible vibration of the solar panels. Meanwhile, the thrusters' plume, which acts on the solar panels, leads to disturbance. Therefore, it is a great challenge to design a controller with high stability, accuracy, and adaptability for the RVD mission of Shenzhou spacecrafts.

Because of the infinite-order feature of the flexible structure, a characteristic model is introduced that uses a second-order linear discrete-time model to describe the dynamics of the flexible structure. Information concerning the high-order feature and nonlinearity is compressed into those characteristic parameters, which are identified online within a convex domain. The detailed steps of characteristic modeling and controller design were given in $\mathrm{Wu}$ et al. (2001). Moreover, other difficulties such as serious disturbance due to thrusters' plume, crosscoupling between attitude and orbit control, and large time delay were taken into account in the process of RVD. The flight data indicate that the high control accuracy of Shenzhou spacecrafts in RVD tasks has reached a high level worldwide (Xie et al., 2013).

\subsection{Data Relay Test Satellite}

The Data Relay Test Satellite (DRTS), which was launched in Sept. 2002 by NASDA, is a research 
communications satellite that demonstrates data relay experiments on the geostationary orbit (Fujiwara et al., 2003). The communication antenna could cause disturbance to the attitude system. Therefore, parameters corresponding to the mass property were estimated online. A feed-forward control was designed based on the estimates (Yamada et al., 2003). The on-orbit flight test showed that the self-tuning adaptive attitude control system has almost the same performance as the one predicted on the ground.

\subsection{Middeck active control experiment}

The middeck active control experiment (MACE) was developed by the Airforce Research Laboratory (AFRL), USA, aimed at the modeling and high-accuracy pointing control issues of flexible structures in the unknown space environment. The experiment was carried out on a test article of $1.7 \mathrm{~m}$ length and $39 \mathrm{~kg}$ weight. It consists of a flexible bus with 17 sensors and 9 actuators including the reaction wheel. There were as many as 50 modes to be controlled, and according to the previous flight experiments, the structural dynamics could change significantly at different modes, which posed a huge challenge to controller design from ground so as to meet the stringent pointing accuracy. This test article was delivered to the orbit by the Space Shuttle flight STS-67 in Mar. 1995. The micro-gravity on-orbit experiment was carried out onboard the Space Shuttle's Middeck to evaluate the identification and controller design. There were two models available: the finite element model and the measurement model. Each of these models contains 130-150 states. The original LQG controller failed to provide satisfactory robustness to parameter uncertainty; therefore, five other modern controllers were developed including the sensitivity-weighted LQG (How et al., 1996), the maximum entropy (How et al., 1996), the multiple model (How et al., 1996), the Popov controller (How et al., 1994), and the $H_{\infty}$ synthesis (Woods-Vedeler and Horta, 1996). The first four controllers were testified on measurement models built using open-loop data, and the last one was validated on-orbit. In the $H_{\infty}$ controller design, the high-order model was reduced using the balanced model reduction technique (Zhou et al., 1996). Multiplicative uncertainty at the output and additive plant uncertainty caused by unmodeled dynamics were considered during the $H_{\infty}$ controller design. The suboptimal $H_{\infty}$ controller design was applied by setting $\gamma=1$ (Section 2.6). According to Woods-Vedeler and Horta (1996), over $50 \mathrm{H}_{\infty}$ controllers were tested on-orbit. The robustness of quite a few closed-loop systems with respect to disturbance was maintained within a satisfactory level. The overall system was stable during the entire flight experiment. Meanwhile, controllers developed upon the reduced-order model showed identical performance to that of the one developed on the full-order model. A ground experiment on Popov control based on the absolute stability theory and $\mathrm{H}_{2}$ analysis was introduced by How et al. (1994). It was demonstrated that, compared to the optimal LQG design, the Popov controller guaranteed superior robust performance.

MACE II is a hands-on experiment aboard the ISS. The ultimate goal is to implement adaptive structural control technology in spacecrafts. The adaptive controller would facilitate controller design on the ground and deal with the change of dynamic characteristics and sensor/actuator failures. MACE II was sent to ISS in 2000 aboard the SPACEHAB module and, after 347 days, it returned aboard STS105. The adaptive structural control methods were able to 'adapt' whenever they sensed changes in vibration or the loss of a sensor or an actuator. These adaptive algorithms provided a decrease in vibration, even when a primary actuator experienced failure (Grocott et al., 1994).

One of the most significant achievements of the MACE program was that it showed the limitations of the traditional fixed-gain control approach and pushed the application of modern control methods in space engineering.

\section{Reentry spacecraft with changing parameters}

The reentry of spacecrafts is another challenging task because of the fast time-varying dynamics, model nonlinearities, and large flight envelopes. In the transonic regime, there would be large aerodynamic coefficient uncertainties. The unstable and partially unknown atmosphere further brings difficulties to the modeling and controller design. Meanwhile, to achieve fast maneuverability, the reentry spacecrafts sometimes contain unstable modes, becoming the most fragile part of the system. 
In this section, several reentry spacecrafts that implemented advanced control methods are introduced. Towards the changing parameters within a large scale, although currently there is no theoretical breakthrough concerning parameter estimation, engineers have proposed several techniques that have been proven effective on some specific spacecrafts. For example, during the reentry of Shenzhou spacecrafts, input-output transformation was developed such that the estimation was carried out within a small scale instead of a large one.

It is worth noting that reentry spacecrafts usually have military backgrounds, and therefore the references are usually limited. Those famous reentry spacecrafts, such as X-37B, are not included here because we could not find published literature with detailed control system design.

\subsection{Italian Unmanned Space Vehicle}

The Italian Aerospace Research Center conducted an aerospace national research program called Unmanned Space Vehicles (USV) in 2003. This program aimed to develop and test new technologies for aerodynamics, guidance, navigation, and control to support the future reusable launch vehicles and aerospace planes. The USV program had been divided into several phases. In the first phase, the focus was on the subsonic, transonic, and low supersonic regimes. Two transonic flight missions, DTFT1 and DTFT2, were scheduled in winter 2007 and spring 2010, respectively. The test vehicle was released from a scientific balloon, which was $20 \mathrm{~km}$ from the ground for DTFT1 and $24 \mathrm{~km}$ from the ground for DTFT2. It then experienced the gliding phase and terminal aero energy management phase subsequentially before landing using a recovery parachute. The DTFT1 flew in a transonic regime with only longitudinal maneuvers. In this relatively mild condition, the conventional control augmentation system with the PID controller was capable of maintaining the angle of an attack at a constant value. However, the DTFT2 was a more complex mission. After releasing from the balloon, it was scheduled to pitch up to reach a predefined angle of an attack and accelerated up to Mach 1.2 at about $15 \mathrm{~km}$. After that, the vehicle had to pull down to keep a constant Mach number. Meanwhile, the vehicle had to select an appropriate landing position out of four preloaded ones online due to the uncontrolled drop position by the balloon. Thus, online trajectory generation and adaptive tracking would be a huge challenge. The high Mach number, rapid maneuvers, and online adaption made the traditional flight control system for DTFT1 short-handed.

A probabilistic robust control synthesis was chosen after being compared to the $\mu$-controller with fuzzy logic gain-scheduling and the direct adaptive model-following controller (Corraro et al., 2011a; 2011b). According to the post-flight data analysis, this robust control law met with great success, and should be capable of controlling the terminal area energy management (TAEM) phase of a reentry vehicle (Corraro et al., 2011b; Nebula and Ariola, 2013).

\subsection{Space Launch System}

For future convenient and reliable access to space including the ISS in low-Earth orbit (LEO), the Moon, Mars, and near-Earth asteroids, NASA has initiated the Space Launch System (SLS) consisting of various exploration-class launch vehicles. The commonly used test vehicle is the F/A-18, the full-scale advanced systems testbed. The features of aerospace vehicles including interactions among control surfaces, control-structure interaction, engine performance, sensor characteristics, and atmospheric behavior are treated specifically. Since 2009, the nonlinear dynamic inversion control and a few adaptive control algorithms have been testified in this Space Launch System, and the flight control system has been validated in many flight tests.

Among the various adaptive control laws, adaptive augmenting control (AAC), which is a special kind of model-reference adaptive control, is probably the most successful one. AAC consists of a classically designed linear controller as the baseline controller (mostly PID) and an adaptive total loop gain. Normally, the adaptive gain stays at the minimum value when the leakage term is not activated. Once the tracking error is too large, the adaptive gain increases immediately to compensate for the performance loss. Most importantly, when an undesirable frequency is detected in the control path, the adaptive gain would decrease until the parasitic dynamics is mitigated. The mathematical representation of AAC is (Orr and VanZwieten, 2012)

$$
\dot{k}_{a}=\left(\frac{k_{\max }-k_{a}}{k_{\max }}\right) a e_{\mathrm{r}}^{2}-\alpha k_{a} y_{s}-\beta\left(k_{T}-1\right),
$$


where $k_{T}=k_{0}+k_{a}, k_{a}$ and $k_{0}$ are the adaptive gain and the minimum gain respectively, $k_{\max }$ is the limit of $k_{a}, a$ is the adaptive error gain, $\alpha$ is the spectral damper gain, $\beta$ is the leakage gain, $e_{\mathrm{r}}$ is the tracking error, and $y_{s}$ is the output of the filter.

This improved model-reference adaptive control finds a balance between the tracking performance and robustness. It was considered a great success when dealing with large pilot inputs, which caused the loss of X-15-3 in 1967 (Thompson and Hunley, 2000).

Both simulations and flight tests were conducted. In the flight test, the F/A-18 flew the SLSlike trajectory and in nominal, off-nominal, and failure scenarios. According to the report of NASA in Jan. 2015, the F/A-18 had flown more than 100 test cases, a few of which lasted more than $60 \mathrm{~min}$. According to the flight results, with AAC on, the system response remained bounded and was capable of dealing with the unstable dynamics (Wall et al., 2015).

Besides, to prevent high-frequency oscillations caused by the high adaptive gain, optimization upon the $L_{2}$-norm of the tracking error was studied by Burken et al. (2010). Neural networks were designed to modify the output of the PI controller toward the optimal criterion. This control system has been simulated in the simulation testbed provided by NASA to show its potential benefits.

\subsection{Shenzhou reentry module}

The Chinese Shenzhou program was authorized in 1992. Since then, Shenzhou 1-10 spacecrafts have been launched in succession. A total of 10 astronauts have been sent into space and returned to ground successfully. The control and guidance technology of the atmospheric entry is a crucial technique in the guidance, navigation, and control (GNC) system of a manned spacecraft. There are several challenges ( $\mathrm{Wu}$ et al., 2009): (1) The reentry module is a small lifting body. This limits the modification capacity of its motion trajectory by changing the lift direction. (2) Environmental parameters such as atmospheric density and wind velocity intensively vary with respect to the altitude of the module. Then the aerodynamic parameters such as drag coefficients and lift-to-drag ratios of the module are changing in a large scale. This will lead to a large landing error if not considered. (3) Some state constraints should be satisfied such that the acceleration during the reentry must remain within a safe limit, the landing site should be in the scheduled area, etc.

For the reentry of Shenzhou's reentry module, a standard reentry trajectory guidance method with an estimated lift-to-drag ratio was proposed ( $\mathrm{Hu}$, 1998). It shares the advantages of both the landing point prediction guidance method and standard trajectory guidance method. The execution steps are as follows: In the transition phase before the reentry, the calculation of landing point prediction and guidance is performed, and a standard reentry trajectory for control is obtained. In the reentry phase, the resulting reentry trajectory is regarded as the guidance goal. A characteristic model-based adaptive method is adopted to estimate the lift-to-drag ratio online, which is used for lift control of the reentry module. To deal with the parameters that vary within a large scale, input-output transformation is developed such that the estimation is then carried out within a small scale instead.

Flight data showed that the guidance and control method could deal with large initial errors, reduce the fuel consumption, and enhance the stability of the reentry. The guidance and control technology for the reentry of Shenzhou spacecrafts was quite mature. The dispersions of the spacecrafts' landing points were all controlled within $13 \mathrm{~km}$.

\subsection{Chang'e 5 test spacecraft}

As the third phase of China's lunar exploration, the Chang'e 5 mission is scheduled to launch in 2017 and is arranged to take samples from the Moon and return to the Earth automatically. To guarantee the success of the mission, China decided to initiate a Chang'e 5 test program to validate the guidance and control of the return capsule from the Moon. Chang'e 5 test vehicle was launched in Oct. 2014 using a CZ-3C rocket. After traveling along the EarthMoon transfer orbit for nearly 8 days, it returned to the Earth on Nov. 1 and landed safely in Inner Mongolia.

After returning from the Moon, the reentry velocity of Chang'e 5 at the $120 \mathrm{~km}$ near-Earth orbit reached the second cosmic velocity. For accurate landing at a low speed, the semi-ballistic skip reentry technique was chosen. This kind of reentry strategy is quite different from that of the Shenzhou reentry module from the near-Earth orbit and has never been used in any of the previous returned capsules 
in China.

For the guidance and control of this semiballistic skip trajectory, a large number of simulations showed that the traditional guidance and control methods cannot provide satisfactory accuracy and reliability, or the capsule may jump back to space and never come back. There is a strong need for an advanced guidance and control technique. Engineers from the Beijing Institute of Control Engineering, China Academy of Space Technology, proposed a first-order all-coefficient adaptive predictioncorrection scheme $(\mathrm{Hu}, 2014)$. This is a simpler form of the commonly used second-order characteristic model-based adaptive control introduced in Section 2. The first-order model showed superior performance to the second-order model in numerical simulations (Hu and Zhang, 2014) and hardware-inthe-loop experiments. Most importantly, this guidance and control algorithm was finally uploaded to the Chang'e 5T test vehicle, and the opening point accuracy was about $500 \mathrm{~m}$ from the expected point.

\section{Spacecraft with high-level require- ments}

In this section, four kinds of spacecraft with different kinds of mission requirements are introduced. The classical PID controller can provide only a system with limited stability margin. Improved mission requirements bring high demands on the robustness and accuracy of the control system, which pushed us to the optimal control methods.

\subsection{Reorientation of the International Space Station}

There are a few ways for the ISS to perform rotation maneuvers while in space. For the shortest path rotation, the cost is high because of the kinematically nonlinear dynamics. When the control moment gyroscopes (CMGs) are inadequate to provide sufficient torques, thrusters are used. Because of CMGs' lifetime issues, momentum desaturation using thrusters is not a wise option. To maintain the CMGs within their operational limits while executing large-angle attitude maneuvers, a practical way is the zero-propellant maneuver (ZPM) method. ZPM generates a rotation trajectory, in which the rotation uses only the naturally occurring environmental torques without reaching the limits of CMGs.
The ZPM attitude control concept was developed at the Draper Laboratory in the mid-1990s. The trajectory for ZPM was solved through an optimal control problem with the constraints of system dynamics, initial and terminal states, and CMGs' capacity. Thanks to the advances in PS methods, which can solve this optimal control problem in an efficient and rapid way, the optimal trajectory can be generated fast and is suitable for engineering applications.

The PS method uses the Lagrange interpolating polynomials over Gaussian nodes to discretize the optimal problem, and ensures a faster convergence rate than the previous fourth-order convergence (Betts and Kolmanovsky, 2002). Meanwhile, by using the covector mapping principle, the PS method allows checking the feasibility and optimality of the optimal solution, which is quite valuable to ZPM. The control loop is a feed-forward open loop, as the carefully designed trajectory requires no thrusters or other external torques.

Two subsequential flight tests were scheduled to testify, for the first time in history, ZPM in Nov. 2006 and Mar. 2007, which reoriented the ISS $90^{\circ}$ and $180^{\circ}$, respectively. The ZPM trajectory was generated a month before the flight date. First, an initial trajectory was generated and tested in simulation. Parameter uncertainties were then considered to improve the robustness of the trajectory. In both flight tests, the trajectories of ZPM were completed successfully and no propellant was used. According to the data provided by the Mission Evaluation Room, the actual attitude ideally fitted the commanded one. According to the report, in the first flight, ZPM saved 50 lbs of propellant, and the second flight saved $100 \mathrm{lbs}$. This was the first time that the PS optimal control theory was ever used in a space mission (Bedrossian et al., 2007; Bedrossian and Bhatt, 2008).

\subsection{Reorientation of the Transition Region and Coronal Explorer}

PS optimal control techniques have been successfully applied to design and implement a minimum-time reorientation maneuver on NASA's space telescope, Transition Region and Coronal Explorer (TRACE), in 2010 (Karpenko et al., 2012). TRACE was designed to document the magnetic features of the solar surface, transition region, and 
corona. The mission requires its attitude control system be able to maintain the pitch and yaw pointing accuracy of 20 arcseconds and to maneuver up to $180^{\circ}$ between targets. Practical optimal control that is suitable for on-orbit implementation is needed to improve the agility of the satellite.

The minimum-time reorientation optimal control by the PS method was first addressed by Proulx and Ross (2001). It could overcome many numerical difficulties associated with finding optimal solutions. Many ground experiments and flight implementations (Ross and Karpenko, 2012) showed the reliability and suitability for generating practical solutions. Besides, most steps in the method were automatic. It could provide the possibility of a fully automated design process for spacecraft operations.

The time-optimal reorientation maneuver of the TRACE spacecraft is obtained by solving the following state-constrained optimal control problem (Karpenko et al., 2012):

$$
B^{\mathrm{R}}:\left\{\begin{array}{cl}
\min _{\boldsymbol{u}} & J(\boldsymbol{x}, \boldsymbol{u}, t)=t_{\mathrm{f}} \\
\text { s.t. } & \dot{\boldsymbol{x}}(t)=\left[\begin{array}{c}
\frac{1}{2} \boldsymbol{Q}(\boldsymbol{\omega}) \boldsymbol{q} \\
\boldsymbol{I}^{-1}(\boldsymbol{\omega} \times \boldsymbol{I} \boldsymbol{\omega}-\boldsymbol{u})
\end{array}\right], \\
& \boldsymbol{x}_{0}=\left[e_{0} \sin \left(\Phi_{0} / 2\right), \cos \left(\Phi_{0} / 2\right), \omega_{0}\right]^{\mathrm{T}}, \\
& \boldsymbol{x}_{\mathrm{f}}=\left[e_{\mathrm{f}} \sin \left(\Phi_{\mathrm{f}} / 2\right), \cos \left(\Phi_{\mathrm{f}} / 2\right), \omega_{\mathrm{f}}\right]^{\mathrm{T}}, \\
& \|\boldsymbol{q}\|_{2}=1, \\
& \left|\omega_{i}\right| \leq \omega_{\max }, i=1,2,3, \\
& \left|u_{i}\right| \leq u_{i, \max }, i=1,2,3 .
\end{array}\right.
$$

The problem aims to find the control $\boldsymbol{u}$ that drives the rigid body from the initial conditions $\boldsymbol{x}_{0}$ at $t=0$ to the final conditions $\boldsymbol{x}_{\mathrm{f}}$ at $t=t_{\mathrm{f}}$, and to minimize the time needed. Denote $\boldsymbol{q}_{0}=\left[e_{0} \sin \left(\Phi_{0} / 2\right), \quad \cos \left(\Phi_{0} / 2\right)\right]^{\mathrm{T}}$ and $\boldsymbol{q}_{\mathrm{f}}=$ $\left[e_{\mathrm{f}} \sin \left(\Phi_{\mathrm{f}} / 2\right), \cos \left(\Phi_{\mathrm{f}} / 2\right)\right]^{\mathrm{T}}$ as the initial and final attitudes of the rigid body, respectively, and $\omega_{0}=\omega_{\mathrm{f}}=0$ are the angular velocities. The state and control for the optimal control problem satisfy the constraints $\|\boldsymbol{q}\|_{2}=1,\left|\omega_{i}\right| \leq \omega_{\max }$, and $\left|u_{i}\right| \leq u_{i, \max }(i=$ $1,2,3)$. The angular velocity must be limited to a certain range to avoid the saturation of the rate gyros, which would lead the satellite out of control.

The object of on-orbit time-optimal control is to perform a reorientation maneuver of $100^{\circ}$. Throughout the maneuver, the body rate was limited within $0.5^{\circ} / \mathrm{s}$ to avoid gyro saturation. The PS-based timeoptimal reorientation maneuver was solved for the spacecraft using the DIDO software (Ross, 2007) and was directly implemented to the onboard attitude control system of the TRACE. The maneuver in the PS-based method was completed in $181.4 \mathrm{~s}$. Moreover, a standard eigenaxis maneuver, which took 205.5 s to complete, was implemented for comparison. Thus, the PS-based time-optimal reorientation was about $12 \%$ faster than the conventional maneuver. More effective usage of the actuators was obtained by building up angular rates around all three body axes. This enabled the TRACE to rotate through a longer path more quickly than the conventional method. The flight results showed evident improvement in agility compared with the conventional method.

\subsection{Fast maneuver of the SSTL microsatellite}

The three-axis attitude control system for microsatellites is based mainly on the PID controller, although the performance of the attitude control hardware has been greatly improved during the last decades. For a given level of energy consumption, the PID controllers limit the attitude response rapidity. On the other hand, to obtain the global optimal feedback control, it is necessary to solve an HJB partial differential equation. Generally, the online calculation of the optimal control for operational attitude control systems is infeasible. The inverse optimal control method was originally developed for this kind of problem (Freeman and Kokotovic, 1996).

The inverse optimal control was originated by Anderson and Moore (1990) to establish certain stable margins for linear systems and was introduced into nonlinear control by Moylan and Anderson (1973). Freeman and Kokotovic (1996) proposed a systematic robust inverse optimal control method that could circumvent the task of solving an HJB equation. The main idea of inverse optimality is based on the fact that the steady-state solution to the HJB equation is a control Lyapunov function obtained from the stabilization problem of the nonlinear system. Theoretically, the solution is globally stable and optimal, and the stability margins can be calculated for the input-to-state stable system.

The inverse optimal controller was validated by a software satellite simulator developed by Surrey Satellite Technology Ltd. (SSTL) for microsatellites (Horri et al., 2011). The simulator incorporates full attitude and orbital dynamics with precise external disturbance, gravity, atmospheric drag, etc. It was 
designed to validate attitude determination and control methods before uploading them to on-orbit satellites. In the experiments, a maneuver from the initial attitude to $30^{\circ}$ off-pointing was first carried out, followed by a maneuver from $30^{\circ}$ off-pointing to the initial attitude, which can be based on either the PD controller with gyro-compensation or an inverse optimal gain scheduled minimum norm controller. The inverse optimal controller was simple to implement and achieved a faster attitude pointing than the PD controller. The settling time was significantly reduced (Horri et al., 2011).

\subsection{Accurate pointing of FASTSAT}

The Fast Affordable Science and Technology Satellite (FASTSAT) (DeKock et al., 2011) is a risktolerant, small-budget microsatellite program. The first FASTSAT is the Huntsville-01 (HSV-01), which was developed collaboratively by the NASA Marshal Space Flight Center, Dynetics, the University of Alabama at Huntsville, and several other industry partners in Huntsville, Alabama. The satellite, whose sides are about 30 inches tall, was launched in Nov. 2010 and operated in the $650 \mathrm{~km}, 72^{\circ}$ inclination orbit.

For a microsatellite like HSV-01, the actuators are magnetic torque rods, which can save a lot of weight and power compared with a wheel-based system. Therefore, the satellite is a roughly periodic system due to the geomagnetic field the satellite flies in.

The scientific experiments assigned to FASTSAT require various attitudes be held. Therefore, the conventional spin-stabilizing method is inappropriate. To achieve high pointing accuracy at different attitudes, the periodic asymptotic LQR controller was chosen. The periodic LQR problem is formalized as

$$
\left\{\begin{array}{cl}
\min _{\boldsymbol{u}} & J=\frac{1}{2} \int_{0}^{T}\left[(\boldsymbol{x}(\tau))^{\mathrm{T}} \boldsymbol{Q} \boldsymbol{x}(\tau)\right. \\
& \left.+(\boldsymbol{u}(\tau))^{\mathrm{T}} \boldsymbol{R} \boldsymbol{u}(\tau)\right] \mathrm{d} \tau+\frac{1}{2}(\boldsymbol{x}(T))^{\mathrm{T}} \boldsymbol{P} \boldsymbol{x}(T) \\
\text { s.t. } & \dot{\boldsymbol{x}}=\boldsymbol{A} \boldsymbol{x}+\boldsymbol{B}(t) \boldsymbol{u}, \\
& \boldsymbol{B}(t)=\boldsymbol{B}(t+T), \\
& \boldsymbol{x}(0) \text { is given. }
\end{array}\right.
$$

The solution to the above problem is $\boldsymbol{u}(t)=$ $-\boldsymbol{R}^{-1}(\boldsymbol{B}(t))^{\mathrm{T}} \boldsymbol{P}(t) \boldsymbol{x}(t)$, where $\boldsymbol{P}(t)$ is periodic with period $T$. To facilitate practical implementation in satellites, the periodic $\boldsymbol{P}(t)$ is approximated by a constant matrix when a series of constraints on matrices $\boldsymbol{R},[\boldsymbol{A}, \boldsymbol{B}]$, and $[\boldsymbol{A}, \boldsymbol{C}]$, as well as the eigenvalues of $\boldsymbol{A}$ are satisfied (Psiaki, 2001).

The periodic LQR implemented onboard FASTSAT was nine-state periodic. According to the flight data that were downlinked, FASTSAT reached a higher pointing accuracy than with the previous best demonstrated local vertical local horizontal relative all-magnetic attitude controller. Meanwhile, it allowed the satellite to point at specific ground targets, instead of using large satellites with complex attitude control systems. It is hoped that this periodic LQR controller can be applied in a nanosatellite platform such as a CubeSat in the future.

\section{Conclusions}

Space activities of humans are becoming frequent with emerging new types of spacecrafts and stringent performance requirements like never before. The traditional PID controller is gradually becoming incapable of meeting the increasing demands. Robust control, adaptive control, and optimal control are undoubtedly the most frequently considered modern control methods in aerospace engineering due to their robustness, adaptability, and optimality. The biggest issue during controller design would be the lack of an accurate mathematical model, and the extremely high-order and nonlinear character of those models. Furthermore, it is extremely difficult to simulate the space environment, which is full of uncertainties and disturbance. How do we guarantee the on-orbit closed-loop performance of the controllers that are developed and tested on the ground? Engineers have been seeking feasible ways to deal with this problem for quite a long time. Meanwhile, rigorous theoretical results in robust and adaptive control are always accompanied by assumptions such as known parameter bounds or appropriate initial values, which have brought troubles to aerospace engineers when designing and tuning those controllers.

Fortunately, aerospace scientists and engineers all over the world have realized the necessity of introducing advanced control to aerospace engineering. A few satellites and reentry vehicles have already experimented with some advanced control laws and have benefited from them. Higher pointing 
accuracy and stronger robustness to disturbances and unstructured dynamics have been validated by some spacecrafts on-orbit with convincing flight data. Motivated by this inspiring trend in aerospace control, we provided a thorough review on the practical applications of advanced control methods in satellites and reentry spacecrafts. Most of these control methods were validated by actual flight experiments, which are particularly valuable to both engineers and scientists. These spacecrafts and their advanced control laws are summarized in Table 1. This table reveals some valuable and interesting disciplines:

\section{Flexibility}

To deal with the flexible modes, engineers from Spacebus 4000, ETS-VI/VIII, and HST reached a consensus on the $\mathrm{H}_{\infty} / \mathrm{H}_{2}$ robust control that provided satisfactory performance on-orbit and on the ground. These successful applications suggested that, when dealing with flexible satellites, the robust control method is usually the first choice.

\section{Uncertainties}

In face of large parameter uncertainties, adaptive control methods, self-tuning and CM-GSAC in particular, outperform other control methods as shown in Shenzhou spacecrafts and DRTS.

\section{Changing parameters}

During the reentry of a spacecraft, a controller with fixed parameters is incapable of dealing with the large variations of parameters. Flight vehicle
F/A-18 has shown the adaptability of MRAC, while Shenzhou and Chang'e 5T spacecrafts have proved that CM-GSAC can deal with the large parameters change during reentry.

Although there have been several successful applications of advanced control laws in aerospace engineering, there is still a long way to go before the new era of aerospace advanced control. Challenges, both theoretical and practical, are brought by the high-level stringent performance requirements in the presence of large flexibility, large parameter changes, structural uncertainties, and high-order modes. Meanwhile, it is indispensable to develop a systematic design process based on modeling, synthesis, verification, and validation. This process should be able to distinguish between controllers and help pick up the optimal ones. To be more specific, such a systematic design process should be carried out through two manifolds, both of which depend highly on spacecraft characteristics and mission requirements.

The first manifold is the modeling of the spacecraft, which should be categorized by the main characters of the spacecraft, such as flexibility or different kinds of uncertainties, and by the priority of mission requirements, such as fast maneuverability or high pointing accuracy.

In the second manifold, controller design should be distinguished among different kinds of plants.

Table 1 Spacecrafts with advanced control methods and the time when they were applied to the corresponding spacecrafts

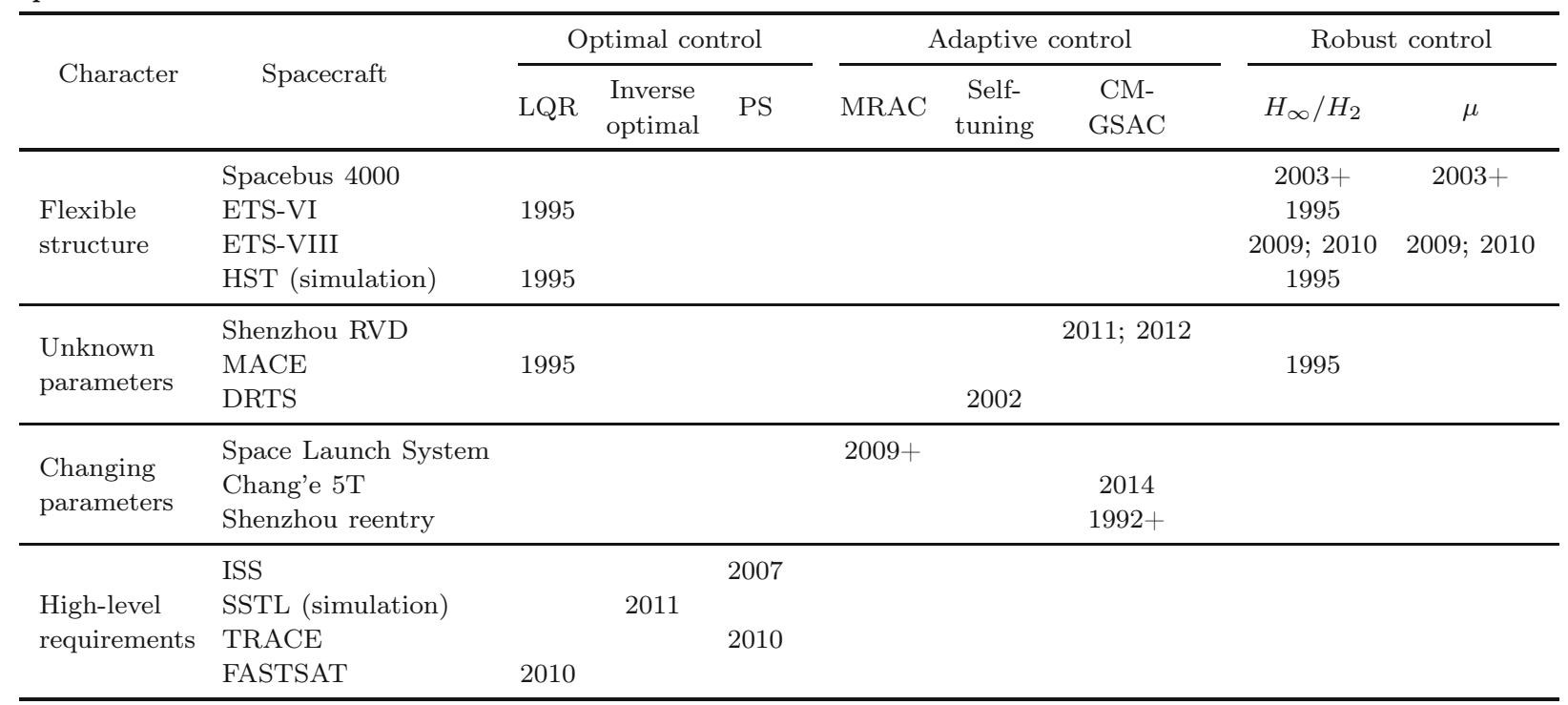


Different control methods are competent with different kinds of models and different control objectives. For instance, optimal control depends highly on the plant models. According to Table 1, this kind of control method performs quite well in spacecrafts with high-level requirements. In particular, the PS method is quite handy in satellite reorientation missions. Robust control allows a limited range of parameter variations, as well as external disturbance. The changing rates of uncertainties could be large as long as they do not exceed the designed bounds. This is the reason why in case of flexible structure, robust control becomes quite useful. On the other hand, adaptive control can identify slowly time-varying parameters that change over a large range. However, its robustness is not quantified. The most prominent field for adaptive control should be the reentry of spacecrafts, where the large parameter variations are beyond the capability of robust control. According to Table 1, it is worth noting that among all the well-known adaptive control methods, the characteristic model-based golden-section adaptive control method has received successful applications in as many as 10 reentry capsules in China. This kind of adaptive control method is different from the general adaptive control method in several ways. First, the characteristic model is built based on a complete analysis of the plant and control objective. Second, its robustness is guaranteed by the golden-section coefficients and the fixed parameter bounds that are calculated beforehand. It is suitable particularly for a system that is slowly time-varying and can be modeled by a second-order or a first-order characteristic model. This is the reason why this simple but effective adaptive control method has served so well in these capsules in China.

We believe that the ultimate goal in aerospace control engineering is a highly automatic system with great intelligence that can deal with all kinds of uncertainties, disturbance, failures, and mission changes on its own. Building a comprehensive database that contains the general models of the representative spacecraft and their candidate advanced controllers is the foundation to this goal. Future artificial intelligence will learn from this database, reason from this knowledge base, and finally evolve to the stage that provides humans with a highly intelligent aerospace control system.

\section{References}

Adachi, S., Yamaguchi, I., Kida, T., et al., 1999. On-orbit system identification experiments on Engineering Test Satellite-VI. Contr. Eng. Pract., 7(7):831-841. http://dx.doi.org/10.1016/S0967-0661(99)00032-5

Anderson, B.D.O., Moore, J.B., 1990. Optimal Control: Linear Quadratic Methods. Prentice Hall, USA.

Anthony, T., Andersen, G., 1995. On-orbit modal identification of the Hubble Space Telescope. Proc. American Control Conf., p.402-406. http://dx.doi.org/10.1109/ACC.1995.529278

Antsaklis, P.J., Michel, A.N., 2007. A Linear Systems Primer. Birkhäuser, Boston.

Åström, K.J., Wittenmark, B., 2008. Adaptive Control (2nd Ed.). Dover Publications Inc., USA.

Bedrossian, N., Bhatt, S., 2008. Space station zero-propellant maneuver guidance trajectories compared to eigenaxis. Proc. American Control Conf., p.4833-4838. http://dx.doi.org/10.1109/ACC.2008.4587259

Bedrossian, N., Bhatt, S., Lammers, M., et al., 2007. First ever flight demonstration of zero propellant maneuver $^{\mathrm{TM}}$ attitude control concept. Proc. AIAA Guidance, Navigation and Control Conf. and Exhibit, p.1-12. http://dx.doi.org/10.2514/6.2007-6734

Betts, J.T., Kolmanovsky, I., 2002. Practical methods for optimal control using nonlinear programming. Appl. Mech. Rev., 55(4):B68. http://dx.doi.org/10.1115/1.1483351

Bharadwaj, S., Osipchuk, M., Mease, K.D., et al., 1998. Geometry and inverse optimality in global attitude stabilization. J. Guid. Contr. Dyn., 21(6):930-939. http://dx.doi.org/10.2514/2.4327

Bukley, A.P., 1995. Hubble Space Telescope pointing control system design improvement study results. J. Guid. Contr. Dyn., 18(2):194-199. http://dx.doi.org/10.2514/3.21369

Burken, J., Nguyen, N., Griffin, B., 2010. Adaptive flight control design with optimal control modification on an F-18 aircraft model. Proc. AIAA Infotech@Aerospace, p.1-17. http://dx.doi.org/10.2514/6.2010-3364

Cao, C.Y., Hovakimyan, N., 2008. Design and analysis of a novel $\mathcal{L}_{1}$ adaptive control architecture with guaranteed transient performance. IEEE Trans. Autom. Contr., $\mathbf{5 3}(2): 586-591$. http://dx.doi.org/10.1109/TAC.2007.914282

Charbonnel, C., 2010. $H_{\infty}$ controller design and $\mu$-analysis: powerful tools for flexible satellite attitude control. Proc. AIAA Guidance, Navigation, and Control Conf., p.1-14. http://dx.doi.org/10.2514/6.2010-7907

Corraro, F., Cuciniello, G., Morani, G., et al., 2011a. Advanced GN\&C technologies for TAEM: flight test results of the Italian Unmanned Space Vehicle. Proc. AIAA Guidance, Navigation, and Control Conf, p.2345-2362.

Corraro, F., Cuciniello, G., Morani, G., 2011b. Flight control strategies for transonic phase of high lift reentry vehicles: comparison and flight testing. Proc. 8th Int. ESA Conf. on Guidance and Navigation Control Systems. 
DeKock, B., Sanders, D., Vanzwieten, T., et al., 2011. Design and integration of an all-magnetic attitude control system for FASTSAT-HSV01's multiple pointing objectives. Proc. 34th Annual Guidance and Control Conf, p.1-19.

Doyle, J., 1984. Lecture Notes in Advanced Multivariable Control. Lecture Note, ONR/Honeywell Workshop, Minneapolis, USA.

Elnagar, G., Kazemi, M.A., Razzaghi, M., 1995. The pseudospectral legendre method for discretizing optimal control problem. IEEE Trans. Autom. Contr., 40(10): 1793-1796. http://dx.doi.org/10.1109/9.467672

Francis, B.A., 1987. A Course in $H_{\infty}$ Control Theory. Springer-Verlag, New York.

Freeman, R.A., Kokotovic, P.V., 1995. Optimal nonlinear controllers for feedback linearizable systems. Proc. American Control Conf., p.2722-2726. http://dx.doi.org/10.1109/ACC.1995.532343

Freeman, R.A., Kokotovic, P.V., 1996. Inverse optimality in robust stabilization. SIAM J. Contr. Optim., 34(4):1365-1391. http://dx.doi.org/10.1137/S0363012993258732

Fujiwara, Y., Nagano, H., Yonechi, H., et al., 2003. The performance of attitude control system on orbit of Data Relay Test Satellite (DRTS). Proc. 21st Int. Communications Satellite Systems Conf. and Exhibit, p.1-8. http://dx.doi.org/10.2514/6.2003-2365

Glover, K., 1984. All optimal Hankel-norm approximations of linear multivariable systems and their $L_{\infty}$ error bounds. Int. J. Contr., 39(6):1115-1193. http://dx.doi.org/10.1080/00207178408933239

Gong, Q., Ross, I.M., Kang, W., et al., 2008. Connections between the covector mapping theorem and convergence of pseudospectral methods for optimal control. Comput. Optim. Appl., 41(3):307-335. http://dx.doi.org/10.1007/s10589-007-9102-4

Grocott, S., How, J., Miller, D., et al., 1994. Robust control design and implementation on the middeck active control experiment. J. Guid. Contr. Dyn., 17(6):11631170. http://dx.doi.org/10.2514/3.21328

Hamada, Y., Ohtani, T., Kida, T., et al., 2011. Synthesis of a linearly interpolated gain scheduling controller for large flexible spacecraft ETS-VIII. Contr. Eng. Pract., 19(6):611-625. http://dx.doi.org/10.1016/j.conengprac.2011.02.005

Hanson, J., 2002. A plan for advanced guidance and control technology for 2nd generation reusable launch vehicles. Proc. AIAA Guidance, Navigation, and Control Conf. and Exhibit, p.1-9. http://dx.doi.org/10.2514/6.2002-4557

Horri, N.M., Palmer, P., Roberts, M., 2011. Design and validation of inverse optimisation software for the attitude control of microsatellites. Acta Astron., 69(11-12):9971006.

http://dx.doi.org/10.1016/j.actaastro.2011.07.010

How, J., Hall, S.R., Haddad, W.M., 1994. Robust controllers for the middeck active control experiment using Popov controller synthesis. IEEE Trans. Contr. Syst. Technol., 2(2):73-87.

http://dx.doi.org/10.1109/87.294331
How, J., Glaese, R., Grocott, S., et al., 1996. Finite element model-based robust controllers for the middeck active control experiment (MACE). IEEE Trans. Contr. Syst. Technol., 5(1):110-118. http://dx.doi.org/10.1109/87.553669

$\mathrm{Hu}$, J., 1998. All coefficients adaptive reentry lifting control of manned spacecraft. J. Astron., 19(1):8-12 (in Chinese).

Hu, J., 2014. Demonstration and Proof of a First-Order Characteristic Model Applied to Prediction-Based AllCoefficient Self-Adaptive Corrector. Technical Report CEK-5T1.LB4, Beijing Institute of Control Engineering (in Chinese).

Hu, J., Zhang, Z., 2014. A study on the reentry guidance for a manned lunar return vehicle. Contr. Theory Appl., 31(12):1678-1685.

Hu, J., Xie, Y.C., Zhang, H., et al., 2011. Shenzhou8 spacecraft guidance navigation and control system and flight result evaluation for rendezvous and docking. Aerosp. Contr. Appl., 37(6):1-5 (in Chinese).

Huang, H., 2015. Multiple characteristic model-based goldensection adaptive control: stability and optimization. Int. J. Adapt. Contr. Signal Process., 29(7):877-904. http://dx.doi.org/10.1002/acs.2510

Karpenko, M., Bhatt, S., Bedrossian, N., et al., 2012. First flight results on time-optimal spacecraft slews. J. Guid. Contr. Dyn., 35(2):367-376. http://dx.doi.org/10.2514/1.54937

Kharisov, E., Gregory, I., Cao, C., et al., 2008. $L_{1}$ adaptive control law for flexible space launch vehicle and proposed plan for flight validation. Proc. AIAA Guidance, Navigation and Control Conf. and Exhibit, p.1-20. http://dx.doi.org/10.2514/6.2008-7128

Kida, T., Yamaguchi, I., Sekiguchi, T., 1997. On-orbit robust control experiment of flexible spacecraft ETSVI. J. Guid. Contr. Dyn., 20(5):865-872. http://dx.doi.org/10.2514/2.4159

Moylan, P., Anderson, B., 1973. Nonlinear regulator theory and an inverse optimal control problem. IEEE Trans. Autom. Contr., 18(5):460-465. http://dx.doi.org/10.1109/TAC.1973.1100365

Nagashio, T., Kida, T., Hamada, Y., et al., $2014 . \quad$ Robust two-degrees-of-freedom attitude controller design and flight test result for Engineering Test Satellite-VIII spacecraft. IEEE Trans. Contr. Syst. Technol., 22(1):157-168. http://dx.doi.org/10.1109/TCST.2013.2248009

Narendra, K.S., Han, Z., 2011. The changing face of adaptive control: the use of multiple models. Ann. Rev. Contr., 335(1):1-12. http://dx.doi.org/10.1016/j.arcontrol.2011.03.010

Nebula, F., Ariola, M., 2013. Italian Unmanned Space Vehicle mission: flight results of the virtual air data algorithm. Proc. 21st Mediterranean Conf. on Control and Automation, p.73-81. http://dx.doi.org/10.1109/MED.2013.6608701

Nurre, G.S., Sharkey, J.P., Nelson, J.D., et al., 1995. Preservicing mission, on-orbit modifications to Hubble Space Telescope pointing control system. J. Guid. Contr. Dyn., 18(2):222-229. http://dx.doi.org/10.2514/3.21373 
Ohtani, T., Hamada, Y., Nagashio, T., et al., 2009. Robust attitude control using $\mu$-synthesis for the large flexible satellite ETS-VIII. J. Space Technol. Sci., 25(1):27-40. http://dx.doi.org/10.11230/jsts.25.1_27

Orr, J., VanZwieten, T.S., 2012. Robust, practical adaptive control for launch vehicles. Proc. AIAA Guidance, Navigation, and Control Conf., p.1-20. http://dx.doi.org/10.2514/6.2012-4549

Paris, S., Riehl, J., Sjauw, W., 2006. Enhanced procedures for direct trajectory optimization using nonlinear programming and implicit integration. Proc. AIAA/AAS Astrodynamics Specialist Conf. and Exhibit, p.1-19. http://dx.doi.org/10.2514/6.2006-6309

Proulx, R., Ross, I.M., 2001. Time-optimal reorientation of asymmetric rigid bodies. Adv. Astronaut. Sci., 109:1207-1227.

Psiaki, M.L., 2001. Magnetic torquer attitude control via asymptotic periodic linear quadratic regulation. $J$. Guid. Contr. Dyn., 24(2):386-394.

http://dx.doi.org/10.2514/2.4723

Ross, I.M., 2005a. A historical introduction to the covector mapping principle. Proc. AAS/AIAA Astrodynamics Specialist Conf., p.1-21.

Ross, I.M., 2005b. A roadmap for optimal control: the right way to commute. Proc. Annual Princeton Conf., p.210-231. http://dx.doi.org/10.1196/annals.1370.015

Ross, I.M., 2007. A Beginner's Guide to DIDO: a MATLAB Application Package for Solving Optimal Control Problems. Available from http://www.elissarglobal.com/ industry/products/.

Ross, I.M., Fahroo, F., 2004. Pseudospectral methods for optimal motion planning of differentially flat systems. IEEE Trans. Autom. Contr., 49(8):1410-1413. http://dx.doi.org/10.1109/TAC.2004.832972

Ross, I.M., Gong, Q., 2010. Emerging Principles in Fast Trajectory Optimization. Course, Naval Postgraduate School, Monterey, CA, USA.

Ross, I.M., Karpenko, M., 2012. A review of pseudospectral optimal control: from theory to flight. Ann. Rev. Contr., 36(2):182-197. http://dx.doi.org/10.1016/j.arcontrol.2012.09.002

Rudin, W., 1975. Functional Analysis. McGraw-Hill, New York, USA.

Scarritt, S., 2008. Nonlinear model reference adaptive control for satellite attitude tracking. Proc. AIAA Guidance, Navigation and Control Conf. and Exhibit, p.1-10. http://dx.doi.org/10.2514/6.2008-7165

Thompson, M.O., Hunley, J.D., 2000. Flight Research: Problems Encountered and What They Should Teach Us. Technical Report NASA/SP-2000-4522, NASA.

Wall, J.H., Miller, C.J., Hanson, C.E., et al., 2015. Inflight suppression of a destabilized F/A-18 structural mode using the space launch system adaptive augmenting control system. Proc. AIAA Guidance, Navigation, and Control Conf., p.1-22.

Woods-Vedeler, J.A., Horta, L.G., 1996. On-Orbit Application of $H_{\infty}$ to the Middeck Active Controls Experiment: Overview of Results. NASA Technical Memorandum 110239.

Wu, H.X., 1990. All Coefficient Adaptive Control Theory and Applications. National Defense Industry Press, Beijing (in Chinese).
Wu, H.X., Liu, Y.W., Liu, Z., et al., 2001. Characteristic modeling and the control of flexible structure. Sci. China Inform. Sci., 44(4):278-291.

Wu, H.X., Hu, J., Xie, Y.C., 2007. Characteristic modelbased all-coefficient adaptive control method and its applications. IEEE Trans. Syst. Man Cybern. Part C, $\mathbf{3 7}(2): 213-221$. http://dx.doi.org/10.1109/TSMCC.2006.887004

Wu, H.X., Hu, J., Xie, Y.C., 2009. Characteristic Model Based Intelligent and Adaptive Control. China Science and Technology Press, Beijing (in Chinese).

Xie, Y.C., Wu, H.X., 1992. The application of the golden section in adaptive robust controller design. Acta Autom. Sin., 18(2):177-185 (in Chinese).

Xie, Y.C., Hu, J., Wang, M., et al., 2013. Accurate and stable control of Shenzhou spacecraft in rendezvous and docking. Proc. 19th IFAC Symp. on Automatic Control in Aerospace, p.524-528.

http://dx.doi.org/10.3182/20130902-5-DE-2040.00063

Yamada, K., Yonechi, H., Wakao, M., et al., 2003. Adaptive attitude control of the data relay test satellite. Proc. 21st Int. Communications Satellite Systems Conf. and Exhibit, p.1-7.

Yu, L., 2002. Robust Control-LMI Method. Tsinghua University, China (in Chinese).

Zhou, K.M., Doyle, J.C., 1999. Essentials of Robust Control. Prentice Hall, New Jersey, USA.

Zhou, K.M., Doyle, J.C., Glover, K., 1996. Robust and Optimal Control. Prentice Hall, Upper Saddle River, New Jersey, USA.

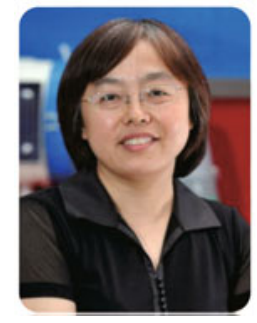

Prof. Yong-chun XIE, first author of this invited review paper, received her B.Sc. in the Department of Electronic Engineering from Tsinghua University in 1989, and received the master and $\mathrm{PhD}$ degrees in automatic control theory and application from Beijing Institute of

Control Engineering, Chinese Academy of Space Technology, in 1991 and 1994, respectively. She is currently Vice Director of the Science and Technology Committee of Beijing Institute of Control Engineering. Prof. Xie has long been engaged in the study of theory, method, and application of spacecraft intelligent adaptive control. Since 2000, she has been responsible for the guidance, navigation, and control of rendezvous and docking, and Shenzhou manned spacecraft GNC system design. She took part in the on-orbit flight control of Shenzhou $\# 8, \# 9, \# 10$, and their rendezvous and docking with Tiangong-1 spacecraft. She has made outstanding contributions to the leaping development of Chinese autonomous rendezvous and docking technology. She has coauthored 1 book, published more than 90 articles, and won 8 national/defense invention patents. Prof. Xie is a member of the editorial committee for Journal of Astronautics and Aerospace Control and Application. 